\section{P242 CO-INFECTIONS WITH CYTOMEGALOVIRUS (CMV) AND SEXUALLY TRANSMITTED INFECTIONS (STIS) IN PREGNANT WOMEN}

${ }^{1}$ Swetha Pinninti*, ${ }^{2}$ Barbara Van Der Pol, ${ }^{2}$ Grace Daniel, 'Karen Fowler, ${ }^{1}$ Suresh Boppana. ${ }^{1}$ University of Alabama at Birmingham, Birmingham, USA; ${ }^{2}$ University of Alabama at Birmingham, Medicine/infectious Diseases, Birmingham, USA

\subsection{6/sextrans-2019-sti.376}

Background Congenital cytomegalovirus infection (cCMV) is a leading cause of hearing loss and neurodevelopmental disabilities. An association between STIs and CMV including higher rates of CMV acquisition and reinfections in women with STIs has been shown in studies of selected populations of women attending STI clinics. However, the interaction between CMV and STIs in the genital tract and whether CMV and STI co-infection increases the risk of intrauterine transmission of CMV remains unclear. The objective of this study is to determine STI prevalence and CMV shedding rates in a cohort of unselected pregnant women.

Methods Vaginal swabs from a cohort of CMV seropositive women in labor from a multi-center study were analyzed. After DNA extraction from vaginal swabs, PCR was performed for detection of CMV, Neisseria gonorrhoeae (GC), Chlamydia trachomatis (CT) and Trichomonas vaginalis (TV). CMV genital shedding rates were compared between groups with and without STIs.

Results Approximately 11\% (11/101)) of pregnant women in this cohort tested positive for STIs (10 with TV and 1 with TV and GC co-infection). None of the women with STIs were shedding $\mathrm{CMV}$ in the genital tract during labor compared to $24 \%(22 / 90)$ of women without STIs. No significant difference in racial distribution was noted between the groups with and without STIs (82\% Black vs 58\% Black respectively; $\mathrm{p}=$ 0.19). Among women shedding CMV in the genital tract, CMV viral load levels ranged from $1.2 \times 102 \mathrm{IU} / \mathrm{ml}-2.3 \times 104$ $\mathrm{IU} / \mathrm{ml}$.

Conclusion In a cohort of unselected pregnant women, none of the CMV seropositive pregnant women with STIs were shedding CMV in the genital tract. Our preliminary findings suggest CMV shedding is not associated with STIs detected late in pregnancy. A larger study is need to confirm these findings and to define the relationship between STIs and cCMV.

Disclosure No significant relationships.

\section{P243 HIGH PREVALENCE OF UREAPLASMA SPP. IN WOMEN VISITING AN STI CLINIC ALTHOUGH NO AZITHROMYCIN RESISTANCE WAS DISCOVERED}

${ }^{1}$ Inge Van Loo*, ${ }^{1}$ Colin Mans, ${ }^{2}$ Christian Hoebe, ${ }^{3}$ Elke Van Westering-Kroon. ${ }^{1}$ Maastricht University Medical Center, Medical Microbiology, Maastricht, Netherlands; ${ }^{2}$ Public Health Service South Limburg, Maastricht University Medical Center (MUMC), Sexual Health, Infectious Diseases and Environmental Health, Medical Microbiology, Care and Public Health Research Institute (CAPHRI), Heerlen, Netherlands; ${ }^{3}$ Maastricht University Medical Center, Pediatrics, Maastricht, Netherlands

\subsection{6/sextrans-2019-sti.377}

Background Ureaplasma spp. are known to reside in the urogenital tract in adults and newborn children. In most cases, Ureaplasma spp. colonization is not harmful to the host. However, Ureaplasma spp. are also known to be involved in non-gonococcal urethritis (NGU) in adults and may also be connected to infertility. In pregnant women, there is speculation that Ureaplasma spp. may cause complications in pregnancy and lead to preterm labor. In preterm newborns Ureaplasma spp. colonization is associated with bronchopulmonary dysplasia. As azithromycin is first choice treatment either in adult as well as in newborn infections resistance to macrolides affects successful therapy. However, little is known about the susceptibility of Ureaplasma spp. In this study we assessed the prevalence of genital Ureaplasma spp. colonization and the prevalence of resistance associated mutations to macrolides.

Methods Genital samples of 512 women visiting our Dutch STI clinic who were routinely screened for Chlamydia trachomatis (CT) and Neisseria gonorrhoeae (NG) were retrospectively tested with Ureaplasma spp. PCR (Amplisens). Of these patients 13\% (66/512) were positive for CT and 1\% (5/512) for NG. Forty five Ureaplasma positive samples were selected to determine mutations that could confer macrolide resistance by sequencing domain $\mathrm{V}$ of the $23 \mathrm{~S}$ RNA gene.

Results In total 78\%(401/512) samples were positive for Ureaplasma spp. Prevalence of Ureaplasma spp. was significantly higher $(\mathrm{p}<0.05)$ among the CT positive samples $88 \%(58 / 66)$ and the NG positive samples $80 \%(4 / 5)$. None of the 45 samples contained mutations associated with macrolide resistance. Conclusion We found a higher prevalence of Ureaplasma spp. in a female patient cohort visiting an STI clinic compared to other studies. However, no resistance associated mutations to macrolides were detected. Higher prevalence of Ureaplasma spp. in STI positive samples underscores its sexually transmitting potential.

Disclosure No significant relationships.

\section{P245 INTEGRATION OF HIV TESTING WITH TUBERCULOSIS AND SEXUALLY TRANSMITTED INFECTIONS AT A TERTIARY CARE HOSPITAL IN DELHI}

Tanisha Bharara*, Renu Gur, Shalini Duggal, Pooja Sharma, Stuti Kaushik, Shilpa Vohra. Dr Baba Saheb Ambedkar Hospital and Medical College, Microbiology, New Delhi, India

\subsection{6/sextrans-2019-sti.378}

Background The National AIDS Control Program (NACP)-IV has been endorsed by our hospital since 2013. Tuberculosis (TB) and Sexually Transmitted Infections (STI) increase the risk of HIV acquisition and vice versa, therefore, NACP- IV, Revised National Tuberculosis Control Program (RNTCP) and National STD control programs were linked in India for better patient outcome.

Methods This is a 3-year retrospective study conducted at Integrated Counseling and Testing Centre (ICTC) of our tertiary care hospital from January 2016 to December 2018. HIV seropositivity among ICTC attendees; HIV-TB and HIV-STI co-infection rates were tested and analyzed as per the National guidelines.

Results A total of 44738 ICTC attendees were tested for HIV, of which 1347 were positive (approximately 3\%). Seropositivity was found to be highest in the reproductive age group (25-49 years). A decline in HIV seropositivity rate was seen, though the number of clients increased by approximately $20 \%$ each year. HIV-TB co-infection rate was found to be $0.18 \%$ in 2016, $0.15 \%$ in 2017 and $0.17 \%$ in 2018 among patients attending DOTS (Directly Observed Treatment Short-Course) centre. HIV positivity among patients referred from STI clinic showed a declining trend from $2.5 \%$ in 2016 to $1.35 \%$ in 2017 and $0.6 \%$ in 2018 . 